\title{
Neutrino Zero Modes and Stability of Electroweak Strings
}

\author{
Dejan Stojkovic \\ Department of Physics \\ Case Western Reserve University \\ Cleveland, OH 44106-7079, USA
}

\begin{abstract}
We discuss massless and massive neutrino zero modes in the background of an electroweak string. We argue that the eventual absence of the neutrino zero mode implies the existence of topologically stable strings where the required non-trivial topology has been induced by the fermionic sector.
\end{abstract}

Recent experimental evidence strongly suggests that neutrinos are very light, but not massless. A simple way to extend the standard model such that it incorporates light neutrino masses is to add a singlet right-handed neutrino. The origin of $M_{R}$ is a vacuum expectation value of some scalar field, acquired at some energy scale much greater than that of the electroweak scale. $M_{R}$ can therefore be taken to be spatially homogeneous. The Lagrangian is:

$$
\begin{aligned}
\mathcal{L}_{S M} & =-\frac{1}{4} W_{\mu \nu}^{a} W^{a \mu \nu}-\frac{1}{4} F_{\mu \nu} F^{\mu \nu}+\left(D_{\mu} \Phi\right)^{\dagger}\left(D^{\mu} \Phi\right)-\lambda\left(\Phi^{\dagger} \Phi-\eta^{2}\right)^{2} \\
& -i \bar{\Psi} \gamma^{\mu} D_{\mu} \Psi-i \overline{e_{R}} \gamma^{\mu} D_{\mu} e_{R}+h^{\prime}\left(\overline{e_{R}} \Phi^{\dagger} \Psi+\bar{\Psi} \Phi e_{R}\right) \\
& +h\left[\bar{\Psi} i \tau_{2} \Phi^{*} \nu_{R}+\overline{\nu_{R}} \Phi^{T}\left(i \tau_{2}\right)^{\dagger} \Psi\right]+\frac{1}{2} \overline{\nu_{R}^{c}} M_{R}^{*} \nu_{R}+\frac{1}{2} \overline{\nu_{R}} M_{R} \nu_{R}^{c}
\end{aligned}
$$

with $\Psi \equiv\left(\nu_{L}, e_{L}^{-}\right)^{T}, \Phi \equiv\left(\phi^{+}, \phi\right)^{T}, \nu_{L}^{T}=(\alpha, \beta,-\alpha,-\beta), \nu_{R}^{T}=(\gamma, \delta, \gamma, \delta), \nu^{c} \equiv C \bar{\nu}^{T}$ and with the usual definition of the gauge and Higgs fields and covariant derivatives [1]. In the Z-string ansatz, all the gauge and Higgs fields are zero except:

$$
Z^{\mu} \sim\left(0,-\frac{v(r)}{r} \vec{e}_{\theta}\right), \quad \phi=\eta f(r) e^{i \theta}
$$

where $v(r)$ and $f(r)$ are the Z-string profile functions. The neutrino equations of motion are: 


$$
\begin{aligned}
i \gamma^{\mu} D_{\mu} \nu_{L} & =h \phi^{*} \nu_{R} \\
i \gamma^{\mu} \partial_{\mu} \nu_{R} & =h \phi \nu_{L}+M_{R}\left(\nu_{R}\right)^{c}
\end{aligned}
$$

Using the ansatz for $\beta, \beta=\sum_{m=-\infty}^{\infty} i \beta_{m}(r) e^{i k_{z} z-i \omega t+i m \theta}$ and similarly for the other spinor components [1], after setting $\omega=k_{z}=0$, we get a set of recursive equations for the coefficients $\beta$ and $\gamma$ :

$$
\begin{aligned}
\beta_{m}^{\prime}+\frac{(m+v)}{r} \beta_{m} & =-h \eta f \gamma_{m} \\
\gamma_{m}^{\prime}-\frac{m}{r} \gamma_{m}-i M_{R} \gamma_{-1-m}^{*} & =-h \eta f \beta_{m}
\end{aligned}
$$

An analytic solution to the system (4) could not be found, but we can learn something about the structure of solutions by looking at their asymptotic behavior. For $m=0$, at large $\mathrm{r}$, where $f \sim 1, v \sim 1$ the system (4) has four solutions for each spinor component. Two of them are exponentially growing while two are exponentially decaying. Only the latter ones are physically acceptable. For small $r$ where, $v \sim v_{0} r^{2}$ and $f \sim f_{0} r$, the structure of the system (4) is such that only three solutions are well behaved i.e. nonsingular and singe valued. With three well-behaved solutions at the origin and two well-behaved solutions at infinity there must be at least one solution which is well-behaved everywhere. Each of the two well-behaved solutions at infinity matches on to a unique linear combination of all four solutions at the origin where only one of them is bad, so there is always one linear combination of the two good solutions at infinity which does not have any contribution from the single bad solution at the origin. We conclude that in this case there is always one well behaved zero mode solution.

In the case of massless neutrino, for large $r, \omega \neq 0$ and $m=0$, we get Bessel's equation for $\beta$,

$$
\beta_{0}^{\prime \prime}+\frac{1}{r} \beta_{0}^{\prime}+\beta_{0}\left(\omega^{2}-\frac{1}{r^{2}}\right)=0
$$

with the general solution given by a linear combination of Bessel's functions:

$$
\beta_{0}(\omega r)=A \sqrt{\omega} J_{1}(\omega r)+B \sqrt{\omega} Y_{1}(\omega r)
$$

where $A$ and $B$ are constants. We see, therefore, that the solution is properly $\delta$-function normalized. In the zero mode limit $\omega \rightarrow 0$. Using the asymptotic expansion for Bessel's function of small argument we get $\beta_{0} \sim 1 / r$ which is the result found in most of the literature. The usual interpretation is that this state is not normalizable. If we look at the solution 
for $\beta_{0}$ as an isolated zero mode, it is obviously not normalizable because the normalization integral diverges logarithmically for large $r$. But, we have seen that this state is actually part of a continuum spectrum of the theory and is Dirac delta function normalizable. Therefore it is a valid zero mode solution. (A similar analysis can be done for zero modes of massive and massless fermions in the background of a domain wall [2].)

In the most general case [3, 4], the presence of a constant left-handed Majorana mass, $M_{L}$, could destroy the neutrino zero mode. The left-handed Majorana mass terms have the form:

$$
\overline{\nu_{L}} M_{L} \nu_{L}^{c}+\overline{\nu_{L}^{c}} M_{L}^{*} \nu_{L}
$$

It was shown in [4, 5] that for a certain range of mass parameters the neutrino zero mode does not exist on a electroweak string. We will argue that this fact imply the existence of the phenomenon which we call "topology induced by fermions".

The possibility of not having a neutrino zero mode while the corresponding electron zero mode is still present is important from the point of view of the string stability under perturbations. In the usual case with a pair of zero modes (see Fig. (四)), perturbations in the Higgs and gauge sectors mix the two zero modes and convert them into two low lying massive states. If the neutrino zero mode is not present, perturbations of the string cannot lift the electron zero mode into a massive mode without violating CP invariance (see Fig. (2)) (the bosonic background is CP invariant since we can absorb all the CP violating phases into the spinor components). This conclusion that a single zero mode can not be removed from the spectrum, obtained from pure symmetry consideration, can be confirmed by studying the stability of fermionic zero modes on electroweak strings under perturbations [6]. For non-topological strings this conclusion seems paradoxical because there is no topology in the bosonic sector that prevents the string from decaying into the vacuum. However, there is no electron zero mode in the vacuum. The only resolution seems to be that the absence of the neutrino zero mode provides topological stability to the string and that the topology enters via the fermionic sector of the model.

It is difficult to incorporate a constant $M_{L}$ into the standard model as a vacuum state. The source of $M_{L}$ could be the vacuum expectation value of an $S U(2)_{L}$ Higgs triplet $\Delta$. The coupling of $\Delta$ to the lepton doublet is given by $\overline{\Psi^{c}} i \tau_{2} \Delta \Psi$. The kinetic terms in the Lagrangian now contain the covariant gradient of the Higgs triplet which couples $\Delta$ to the Zgauge field. This coupling forces a solution of the bosonic equations of motion for $\Delta$ to wind around the Z-string and hence to vanish on the string axis. Similarly, a cubic interaction term $\Phi^{\dagger} i \tau_{2} \Delta \Phi^{*}$ forces the triplet to wind due to $\theta$ dependence of $\Phi$. The way to avoid the problem of coupling to the gauge fields is to set all the standard model gauge fields to zero. In this case the gauge groups become global. We can also set a cubic interaction term $\Phi^{\dagger} i \tau_{2} \Delta \Phi^{*}$ to zero. This can be done by requiring the lepton number conservation in 


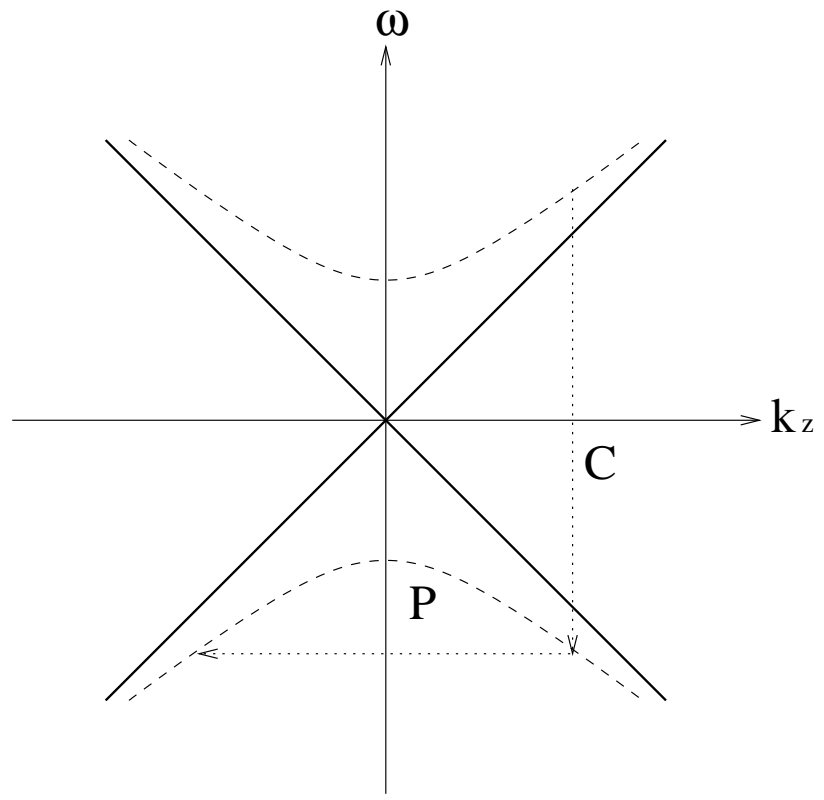

Figure 1: In the usual case, the fermionic spectrum contains two lines (solid) that describe zero modes boosted in the $z$-direction. One line describes the left-moving and the other the right-moving zero modes. Perturbations of the bosonic background can mix the two zero modes at the origin and convert them into two massive modes that lie on hyperbolae. The points on the two hyperbolae are related by CP transformations as shown.

the initial Lagrangian. If we consider the situation in which the doublet and the triplet get vacuum expectation values:

$$
\Phi=\left(\begin{array}{c}
0 \\
\eta f(r) e^{i \theta}
\end{array}\right), \Delta=\left(\begin{array}{cc}
0 & 0 \\
M_{L} & 0
\end{array}\right)
$$

the symmetry breaking pattern is $S U(2)_{L} \times U(1)_{Y} \rightarrow U(1)_{Q}$ where all the symmetry groups are global. The model, although not the standard model anymore, supports a spatially homogeneous $M_{L}$ and the non-topological global Z-string, and the above discussion applies to it.

From the other point of view, if we want to keep all the features of the standard model, at a given moment of time, one can consider fermions with the general mass matrix in the background of the standard model Z-string. We again consider the symmetry breaking pattern (8). As is characteristic for a phase transition phenomenon, the Higgs and gauge fields, initially take different random configurations at different regions in space. One of the possible configurations we would like to analyze is a configuration in which $\phi$ winds 


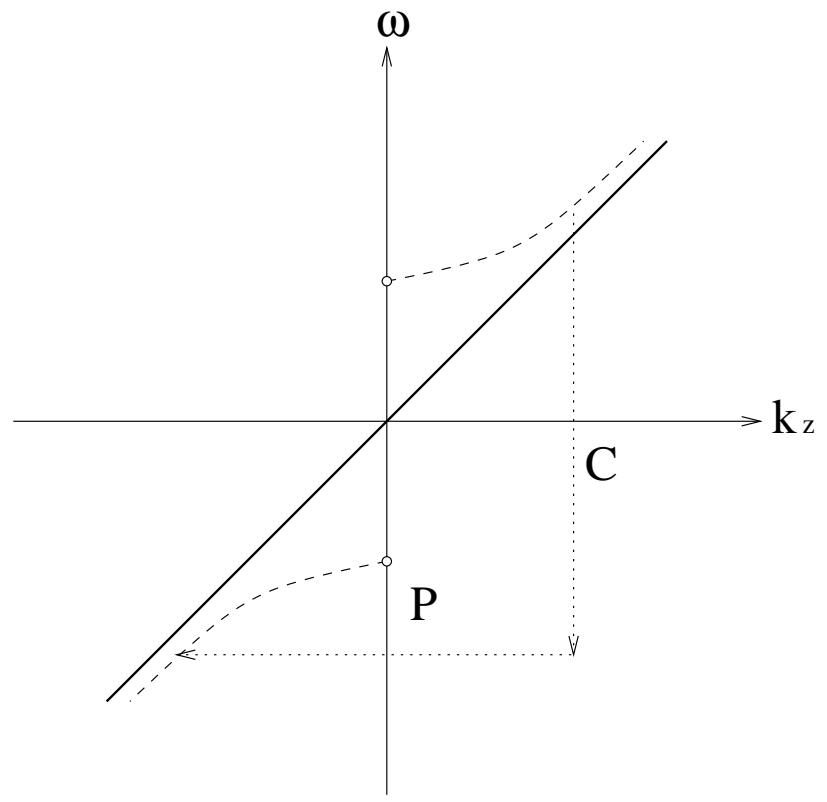

Figure 2: In the present case, the spectrum contains only the line of boosted electron zero modes (the $\omega=+k_{z}$ line). Perturbations of the background cannot change the line into the spectrum for massive fermions (hyperbolae). In particular, perturbations cannot convert the single zero mode $\left(\omega=0=k_{z}\right)$ into a massive mode because $\mathrm{CP}$ invariance would require it to split into two modes, leading to a discontinuous spectrum.

around some axis while $M_{L}$ and $M_{R}$ are constant. If a non-trivial topology was present in the bosonic sector of the model, such a configuration would be stable. However the present model does not have any non-trivial bosonic topology. A configuration with the constant $M_{L}$ is not a solution of the equations of motion and does not minimize the energy. Usually, such a non-equilibrium configuration evolves continuously into a state of minimum energy which, in this case, is the vacuum where all the fields are constant, unless there are topological sectors which forbid the evolution from one sector to another. The bosonic sector of the standard model does not contain such topological sectors. However, if the parameters of the model are such that there is no neutrino zero mode [4, 5], then the constant $M_{L}$ configuration supports a single electron zero mode. As we have argued, CP invariance forbids removing (or creating) a single zero mode and different configurations can not continuously transform into one another.

Although we did not show that there is a topological charge density which can be associated with this "fermionic topology", we showed that there is an integer $n_{L}-n_{R}$ (the difference between the left- and right- moving fermionic zero modes, namely electron and 
neutrino zero modes) that characterizes the field configuration. We argue that $n_{L}-n_{R}$ plays the role of the topological charge and cannot change under continuous evolution of the fields. Since $n_{L}-n_{R}$ in the vacuum is zero while in our chosen background is 1 , the background and the vacuum are in distinct topological sectors. Thus, the configuration with a single electron zero mode leads to a stable string where the non-trivial topology arises from the fermionic sector of the theory and not, as is usual, from the non-trivial topology associated with the bosonic vacuum manifold.

A real challenge would be a construction of an index theorem describing this phenomenon. In this case, there should exist a topological charge density associated with this "fermionic topology". Unlike the all known versions of (topological) index theorems which take only the bosonic field configurations into account, this new index theorem must also depend on the fermionic sector of the theory.

\section{References}

[1] Glenn D. Starkman, Dejan Stojkovic, Tanmay Vachaspati, Neutrino Zero Modes on Electroweak Strings, Phys. Rev. D63, 085011 (2001)

[2] Dejan Stojkovic, Fermionic Zero Modes on Domain Walls, Phys. Rev. D63, 025010 (2001)

[3] Glenn D. Starkman, Dejan Stojkovic, Neutrino Masses and Mixings with General Mass Matrices, Phys. Lett. B480 381-391, (2000)

[4] Glenn Starkman, Dejan Stojkovic, Zero Modes on Z-strings with General Mass Matrix, In Preparation

[5] Glenn Starkman, Dejan Stojkovic, Tanmay Vachaspati, Topology from Fermions, (hepth/0103039)

[6] Dejan Stojkovic, Neutrino Mass and its Implications for the Zero Mode and Vacuum Structures of the Standard Model and its Extensions, Ph.D. Thesis (2001) 